\title{
HYBRID SPACES WITH INTERESTING COHOMOLOGY
}

\author{
KATHRYN LESH
}

\begin{abstract}
Let $p$ be an odd prime, and let $R$ be a polynomial algebra over the Steenrod algebra with generators in dimensions prime to $p$. To such an algebra is associated a $p$-adic pseudoreflection group $W$, and we assume that $W$ is of order prime to $p$ and irreducible. Adjoin to $R$ a one-dimensional element $z$, and give $R[z]$ an action of the Steenrod algebra by $\beta z=0$ and $\beta x=$ $(|x| / 2) z x$ for an even dimensional element $x$. We show that the subalgebra of elements of $R[z]$ consisting of elements of degree greater than one is realized uniquely, up to homotopy, as the cohomology of a $p$-complete space. This space can be thought of as a cross between spaces studied by Aguade, Broto, and Notbohm, and the Clark-Ewing examples, further studied by Dwyer, Miller, and Wilkerson.
\end{abstract}

\section{INTRODUCTION}

In this paper we add to the collection of algebras over the Steenrod algebra which are known to be uniquely realized as the mod $p$ cohomology of $p$ complete spaces. We construct hybrids of spaces constructed by Aguade, Broto, and Notbohm [ABN] and of spaces constructed by Clark and Ewing [C-E], further studied by Dwyer, Miller, and Wilkerson [DMW]. To describe our results, we let $p$ be an odd prime, and let $R$ be a polynomial algebra over the Steenrod algebra with generators in dimensions prime to $p$. It was shown in [DMW, Theorem 1.2 and Corollary 1.3] that such algebras $R$ are in one-to-one correspondence with $p$-adic pseudoreflection groups $W \subseteq G L_{n}\left(Z_{p}\right)$ of order prime to $p$, and that such an $R$ is realized uniquely (up to homotopy) as the cohomology of a $p$-complete space by the Borel construction $E W \times_{W} K\left(Z_{p}^{n}, 2\right)$ for its associated pseudoreflection group.

Now consider the algebra over the Steenrod algebra obtained by taking a polynomial algebra $R$ as above and adjoining an element $z$ in dimension one. Impose an action of the Steenrod algebra on $R[z]$ by setting $\beta z=0$ and $\beta x=(|x| / 2) z x$ for an even dimensional element $x$, and extending by the Cartan formula. Let $A_{W}$ denote the subalgebra of $R[z]$ consisting of elements of degree greater than one.

Theorem 1.1. Let $p$ be an odd prime, and let $W$ be an irreducible finite pseudoreflection subgroup of $G L_{n}\left(Z_{p}\right)$ of order prime to $p$. There is, up to homotopy, a unique $p$-complete space $X_{W}$ with $H^{*}\left(X_{W} ; Z / p\right) \cong \mathbf{A}_{W}$. That is, if $Y$ is a

Received by the editors October 1, 1993.

1991 Mathematics Subject Classification. Primary 55P15; Secondary 55P60. 
p-complete space and $H^{*}(Y ; Z / p) \cong \mathbf{A}_{W}$, then there is a homotopy equivalence $X_{W} \rightarrow Y$.

We note that if $A_{W}$ has one polynomial generator in dimension $2 r$ prime to $p$, we obtain the "interesting cohomology" algebra $A_{r} \cong \mathbf{F}_{p}\left[x_{2 r}\right] \otimes E\left(\beta x_{2 r}\right)$ of $[\mathrm{ABN}]$, and hence this work is a generalization of the corresponding theorem of $[\mathrm{ABN}]$.

The organization of the paper is as follows. In Section 2, we construct the spaces $X_{W}$ with $H^{*} X_{W} \cong A_{W}$. In the rest of the paper, we consider a $p$ complete space $Y$ with $H^{*} Y \cong A_{W}$ and construct a homotopy equivalence $X_{W} \rightarrow Y$. Let $V$ be an elementary abelian $p$-group whose rank is equal to the number of polynomial generators in $A_{W}$. Our strategy, like that of [ABN], is to study a component of the mapping space $\operatorname{map}(B V, Y)$. In Section 3, we compute Lannes's $T$-functor on $A_{W}$ as a first step to understanding map $(B V, Y)$, and in Section 4 we show that $T$ gives the actual cohomology of the mapping space for a component we are interested in. Section 5 considers the homotopy theoretic properties of the mapping space and uses the basepoint evaluation $\operatorname{map}(B V, Y) \rightarrow Y$ to construct a homotopy equivalence $X_{W} \rightarrow Y$. We note that the irreducibility of $W$ is required only in the proof of Proposition 5.1.

The following conventions and notation will be in effect throughout the paper. The prime $p$ is odd, and cohomology is taken with $\bmod p$ coefficients unless otherwise specified. We write $\mathscr{K}$ for the category of unstable algebras over the Steenrod algebra. If $X$ is a space with an action of the group $G$, we write $\operatorname{Borel}(G, X)$ for the Borel construction $E G \times_{G} X$. Following the notation of [DMW], we write $\hat{B} T$ for the space $K\left(Z_{p}^{n}, 2\right)$, where $n$ will always be clear from context. For one last piece of notation, recall that for odd primes $p$, $G L_{1}\left(Z_{p}\right) \cong Z /(p-1) \times Z_{p}$. We write $\pi$ for the discrete group $Z_{p} \subseteq G L_{1}\left(Z_{p}\right)$ under the fixed choice of splitting $x \rightarrow(1+p)^{x}$.

\section{Bokel Constructions}

We begin our construction by considering Borel constructions analogous to those of [ABN, Section 5]. Let $\pi \subseteq \operatorname{Aut}\left(Z_{p}\right)$ act diagonally on each component of $Z_{p}^{n}$, i.e. let $\pi$ be embedded in $G L_{n}\left(Z_{p}\right)$ as multiples of the identity matrix. There is a corresponding action of $\pi$ on $\hat{B} T=K\left(Z_{p}^{n}, 2\right)$, and we begin with the Borel construction for this action.

Proposition 2.1. $H^{*} \operatorname{Borel}(\pi, \hat{B} T) \cong E(z) \otimes \mathbf{F}_{p}\left[x_{1}, \ldots, x_{n}\right]$ where $|z|=1$, $\left|x_{i}\right|=2$, and $\beta x_{i}=z x_{i}$.

Proof. We apply the Serre spectral sequence to the fibration

$$
\hat{B} T \rightarrow \operatorname{Borel}(\pi, \hat{B} T) \rightarrow B \pi .
$$

The action of the fundamental group of the base on the mod $p$ cohomology of the fiber is trivial, since $1 \in \pi$ acts on each coordinate of $Z_{p}^{n}$ by multiplication by $1+p$. The $E_{2}$-term of the spectral sequence is $E(z) \otimes \mathbf{F}_{p}\left[x_{1}, \ldots, x_{n}\right]$ where $|z|=1,\left|x_{i}\right|=2$. For dimensional reasons there are no differentials, so $E_{2}=$ $E_{\infty}$. No multiplicative extensions are possible, and it remains only to compute $\beta x_{i}$. 
To do this, we reduce to the one-dimensional case. Consider the fibration

$$
K\left(Z_{p}, 2\right) \rightarrow \operatorname{Borel}\left(\pi, K\left(Z_{p}, 2\right)\right) \rightarrow B \pi
$$

which, by the same reasoning as above, has Serre spectral sequence $E_{2}=E_{\infty}=$ $E\left(z^{\prime}\right) \otimes \mathbf{F}_{p}[x]$. To compute the action of the Bockstein, we compute low dimensional $Z_{p}$ cohomology groups of $\operatorname{Borel}\left(\pi, K\left(Z_{p}, 2\right)\right)$, using the Serre spectral sequence with twisted coefficients. For this spectral sequence, the $E_{2}$-term is

$$
E_{2}^{p, q}=H^{p}\left(\pi ; H^{q} K\left(Z_{p}, 2\right)\right),
$$

which is zero if $p>1$. The only possible contribution to the cohomology in dimension 2 comes from $H^{0}\left(\pi ; H^{2} K\left(Z_{p}, 2\right)\right)$, which is zero. Contributing to cohomology in dimension 3 we have only $H^{1}\left(\pi ; H^{2} K\left(Z_{p}, 2\right)\right)=Z / p$. Hence in the mod $p$ cohomology of $\operatorname{Borel}\left(\pi, K\left(Z_{p}, 2\right)\right)$ we must have $\beta x=z^{\prime} x$. But now the proposition follows easily by considering a map of fibrations

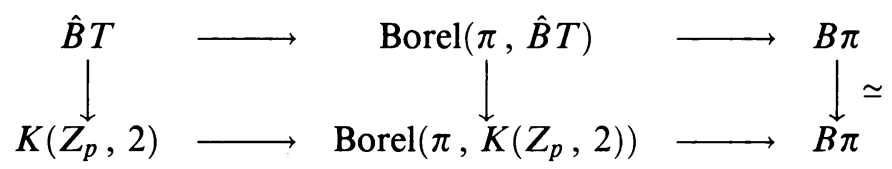

which maps $z^{\prime}$ to $z$ and $x$ to $x_{i}$.

For the next stage of our construction, we will study Borel constructions for actions of subgroups of $G L_{n}\left(Z_{p}\right)$ on $K\left(Z_{p}^{n}, 2\right)=\hat{B} T$. The subgroups we will be interested in are of the form $W \times \pi$, where $W$ is a finite pseudoreflection subgroup of $G L_{n}\left(Z_{p}\right)$ of order prime to $p$, and $\pi$, as before, is embedded in $G L_{n}\left(Z_{p}\right)$ as scalar matrices.

Proposition 2.2. If $W \subseteq G L_{n}\left(Z_{p}\right)$ and $(|W|, p)=1$, then

$$
H^{*} \operatorname{Borel}(W \times \pi, \hat{B} T) \cong E(z) \otimes \mathbf{F}_{p}\left[x_{1}, \ldots, x_{n}\right]^{W} .
$$

Proof. Since $\operatorname{Borel}(W \times \pi, \hat{B} T) \simeq \operatorname{Borel}(W, \operatorname{Borel}(\pi, \hat{B} T))$ and because $(|W|, p)=1$, we know that $H^{*} \operatorname{Borel}(W \times \pi, \hat{B} T)$ is isomorphic to the invariants of the $W$ action on $H^{*} \operatorname{Borel}(\pi, \hat{B} T)$. Now the actions of $W$ and $\pi$ on $\hat{B} T$ commute, so the inclusion of the fiber

$$
\hat{B} T \rightarrow \operatorname{Borel}(\pi, \hat{B} T),
$$

is $W$-equivariant. It is also an isomorphism on even dimensional cohomology, so the action of $W$ on the even dimensional cohomology of $\operatorname{Borel}(\pi, \hat{B} T)$ is the same as that on $H^{*} \hat{B} T$. Again because the actions of $W$ and $Z_{p}$ on $\hat{B} T$ commute, we see that $W$ fixes the one-skeleton of $\operatorname{Borel}(\pi, \hat{B} T)$, and hence fixes the one-dimensional cohomology class $z$.

We are now ready to construct the spaces for which we claim homotopical uniqueness. Notice that the algebra over the Steenrod algebra formed by the elements of $H^{*} \operatorname{Borel}(W \times \pi, \hat{B} T)$ of degree greater than or equal to two is isomorphic to $A_{W}$-this is because a polynomial algebra over the Steenrod algebra which has generators in dimensions prime to $p$ is isomorphic to the invariants of the action of its associated pseudoreflection group $W \subseteq G L_{n}\left(Z_{p}\right)$ on $H^{*} \hat{B} T$. Further, Proposition 2.1 shows that the action of the Bockstein on 
$H^{*} \operatorname{Borel}(W \times \pi, \hat{B} T)$ is the same as the action on $A_{W}$. We can realize the algebra $A_{W}$ as the cohomology of a space as follows. Since

$$
\pi_{1} \operatorname{Borel}(W \times \pi, \hat{B} T) \cong W \times \pi,
$$

we can take a homotopy element

$$
f: S^{1} \rightarrow \operatorname{Borel}(W \times \pi, \hat{B} T)
$$

representing $1 \in \pi$, and note it is an isomorphism on $H^{1}$. Letting $C_{W}$ denote the cofiber of $f$, we have $H^{*} C_{W} \cong A_{W}$. Let $X_{W}$ be the $p$-completion of $C_{W}$.

Proposition 2.3. $X_{W}$ is a simply connected, p-complete space with $H^{*} X_{W} \cong$ $A_{W}$. The homotopy groups of $X_{W}$ are finite p-groups.

Proof. Since $H_{1}\left(C_{W} ; \mathbf{F}_{p}\right)$ is zero, $C_{W}$ is $p$-good (has the same mod $p$ homology as its $p$-completion) and has simply connected $p$-completion by [B-K, VII, 3.1, 3.2]. The finiteness of the homotopy, which will be needed for the proofs of Section 4, follows exactly as in [ABN, Thm 5.5] from knowing the cohomology of $X_{W}$. We summarize the argument, which relies on the fact that $X_{W}$ is simply connected and $p$-complete by [B-K, I, Proposition 5.2] . First, by [ABN, Proposition 5.7], the fact that $H^{*}\left(X_{W} ; \mathbf{F}_{p}\right)$ is of finite type implies that $H^{*}\left(X_{W} ; Z_{p}\right)$ is a finitely generated $Z_{p}$-module. Next, standard properties of the Bockstein spectral sequence show that the $E_{\infty}$ term of the Bockstein spectral sequence for $X_{W}$ is zero. Because $H^{*}\left(X_{W} ; Z_{p}\right)$ is known to be finitely generated in each dimension, this tells us that in fact $H^{*}\left(X_{W} ; Z_{p}\right)$ consists of finite $p$-groups. Then a Serre class argument shows that $\pi_{*} X_{W}$ also consists of finite $p$-groups.

\section{Computations With $T$}

Let $Y$ be a $p$-complete space with $H^{*} Y \cong A_{W}$ and let $V$ be an elementary abelian $p$-group with rank equal to the number of polynomial generators of $A_{W}$. In this section and the next we compute the cohomology of a component of the mapping space $\operatorname{map}(B V, Y)$ by using Lannes's $T^{V}$ functor. This section computes a component of $T^{V} A_{W}$.

First we establish some notation. Choose an identification of $H^{*} \hat{B} T$ with a polynomial subalgebra of $H^{*} B V$ and write $f: H^{*} \operatorname{Borel}(\pi, \hat{B} T) \rightarrow H^{*} B V$ for the map that is this identification on even dimensions and is zero on odd ones. We write $f$ also for the restriction to $A_{W} \subseteq H^{*} \operatorname{Borel}(\pi, \hat{B} T)$, and note that if we let $W$ act on $H^{*} B V$ by first reducing $W$ to $G L(V)$, then $f$ restricted to $A_{W}$ is inclusion of $W$-invariants on even dimensions (and zero on odd ones). If $H^{*} Y \cong A_{W}$ then $H^{*} Y$ is of finite type, and there is an isomorphism $[B V, Y] \cong \operatorname{Hom}_{\mathscr{K}}\left(H^{*} Y, H^{*} B V\right)=\operatorname{Hom}_{\mathscr{H}}\left(A_{W}, H^{*} B V\right)$ [Lannes, Theorem 0.4]. By abuse of notation, we write $\operatorname{map}(B V, Y)_{f}$ for the component of the mapping space which corresponds to $f: A_{W} \rightarrow H^{*} B V$.

We begin the calculation of $H^{*} \operatorname{map}(B V, Y)_{f}$ in this section with the computation of Lannes's $T$-functor. Recall that given an elementary abelian $p$-group $V$, the functor $T^{V}: \mathscr{K} \rightarrow \mathscr{K}$ is left adjoint to the functor $H^{*} B V \otimes-$. The 
evaluation function

$$
B V \times \operatorname{map}(B V, Y) \rightarrow Y
$$

induces a map on cohomology

$$
H^{*} Y \rightarrow H^{*} B V \otimes H^{*} \operatorname{map}(B V, Y),
$$

the adjoint to which is a map

$$
\lambda: T^{V} H^{*} Y \rightarrow H^{*} \operatorname{map}(B V, Y) .
$$

Under favorable circumstances, $\lambda$ is an isomorphism, and so the computation of $T^{V} H^{*} Y$ is a natural first step in the study of $\operatorname{map}(B V, Y)$. Further, because $T^{V} K$ is a functor of $V$ as well as of $K$, there is an action of $G L(V)$ on $T^{V} K$. For an appropriate model of $B V$, there is likewise a $G L(V)$ action on $\operatorname{map}(B V, Y)$, and it is not hard to show that $\lambda: T^{V} H^{*} Y \rightarrow H^{*} \operatorname{map}(B V, Y)$ is $G L(V)$-equivariant. In our calculation of $T^{V} A_{W}$ we will also compute part of the $G L(V)$ action, which is required for later application in Section 5.

Since we are only interested in one component of the mapping space, namely $\operatorname{map}(B V, Y)_{f}$, we restrict our attention to the correponding component of $T^{V} H^{*} Y$. Given the map $f: H^{*} Y \rightarrow H^{*} B V$, the adjoint map $T^{V} H^{*} Y \rightarrow$ $\mathbf{F}_{p}$ gives an action of $\left(T^{V} H^{*} Y\right)^{0}$ on $\mathbf{F}_{p}$. We define the component of $T$ corresponding to $f$ by

$$
T_{f}^{V} H^{*} Y \cong T^{V} H^{*} Y \otimes_{\left(T^{V} H^{*} Y\right)^{0}} \mathbf{F}_{p},
$$

and $\lambda$ induces a map

$$
T_{f}^{V} H^{*} Y \rightarrow H^{*} \operatorname{map}(B V, Y)_{f} .
$$

Now $T^{V} H^{*} Y$ and $\operatorname{map}(B V, Y)$ both come equipped with an action of $W \subseteq$ $G L_{n}\left(Z_{p}\right)$ by first reducing $W$ to $\bar{W} \subseteq G L(V)$. In the case that $H^{*} Y \cong A_{W}$ and $f$ is inclusion of invariants on even dimensions, the $W$ action on $H^{*} B V$ fixes $f$, and hence the action of $W$ on $T^{V} A_{W}$ restricts to an action on $T_{f}^{V} A_{W}$. Our goal in this section is to compute $T_{f}^{V} A_{W}$ as a $W$-module. We do this in two stages, first computing $T^{V} H^{*} \operatorname{Borel}(\pi, \hat{B} T)$ by a geometric argument, and then retrieving $T_{f}^{V} A_{W}$ from it by algebraic arguments relying on the fact that $A_{W} \subseteq H^{*} \operatorname{Borel}(\pi, \hat{B} T)$ and $T^{V}$ is exact.

To compute $T^{V} H^{*} \operatorname{Borel}(\pi, \hat{B} T)$, we will actually run Lannes's machinery backwards. We first compute the cohomology of the space

$$
\operatorname{map}(B V, \operatorname{Borel}(\pi, \hat{B} T)),
$$

and then we check that the hypotheses of the following lemma, specialized from one of Lannes, are satisfied:

Lemma 3.1. [Lannes, Proposition 3.4.4] Let $Z$ be a fibrant p-complete space whose mod $p$ cohomology is of finite type, and let $S$ be a closed subset of $\operatorname{Hom}_{\mathscr{H}}\left(H^{*} Z, H^{*} B V\right)$. If $H^{*} \operatorname{map}(B V, Z)_{S}$ is of finite type and $\operatorname{map}(B V, Z)_{S}$ is p-complete, then

$$
\lambda: T_{S}^{V} H^{*} Z \rightarrow H^{*} \operatorname{map}(B V, Z)_{S}
$$

is an isomorphism. 
This will allow us to compute $T^{V} H^{*} \operatorname{Borel}(\pi, \hat{B} T)$.

Proposition 3.2.

$$
H^{*} \operatorname{map}(B V, \operatorname{Borel}(\pi, \hat{B} T)) \cong \bigoplus_{\operatorname{End}(V)} H^{*} \operatorname{Borel}(\pi, \hat{B} T) .
$$

Proof. The key ingredient is the fact that $\operatorname{map}(B V, \hat{B} T) \simeq \coprod_{\operatorname{End}(V)} \hat{B} T$ (see, for example, [D-Z, Theorem 1.1]). The space $\operatorname{Borel}(\pi, \hat{B} T)$ sits in a fibration

$$
\hat{B} T \rightarrow \operatorname{Borel}(\pi, \hat{B} T) \rightarrow B \pi,
$$

which gives rise to the fibration

$$
\operatorname{map}(B V, \hat{B} T) \rightarrow \operatorname{map}(B V, \operatorname{Borel}(\pi, \hat{B} T)) \rightarrow \operatorname{map}(B V, B \pi) .
$$

There is a continuous function

$$
\operatorname{Borel}(\pi, \operatorname{map}(B V, \hat{B} T)) \rightarrow \operatorname{map}(B V, \operatorname{Borel}(\pi, \hat{B} T))
$$

which takes $(e, g: x \rightarrow y)$ to $\bar{g}: x \rightarrow(e, y)$ and fits into a map of fibrations:

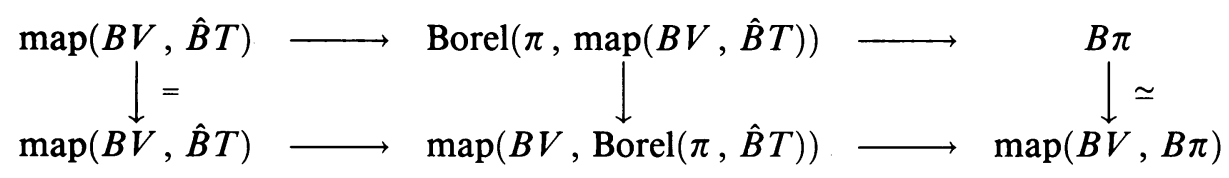

One can see that the right vertical map is an equivalence by showing that it is an isomorphism on homotopy, using the fact that $B \pi$ is an Eilenberg-Mac Lane space. Another easy check shows that the left arrow is actually the identity, so the center vertical map is an equivalence. Since $\operatorname{map}(B V, \hat{B} T) \simeq \bigsqcup_{\operatorname{End}(V)} \hat{B} T$, and $\pi$ can be seen to act diagonally on the components, we see that in fact

$$
\operatorname{map}(B V, \operatorname{Borel}(\pi, \hat{B} T)) \simeq \operatorname{Borel}(\pi, \operatorname{map}(B V, \hat{B} T)) \simeq \coprod_{\operatorname{End}(V)} \operatorname{Borel}(\pi, \hat{B} T) .
$$

\section{Corollary 3.3.}

$$
T^{V} H^{*} \operatorname{Borel}(\pi, \hat{B} T) \cong \bigoplus_{\operatorname{End}(V)} H^{*} \operatorname{Borel}(\pi, \hat{B} T)
$$

Proof. We must check the hypotheses of Lemma 3.1. From Proposition 3.2 we know that $H^{*} \operatorname{map}(B V, \operatorname{Borel}(\pi, \hat{B} T))$ is of finite type, so we need only check that $\operatorname{Borel}(\pi, \hat{B} T)$ and $\operatorname{map}(B V, \operatorname{Borel}(\pi, \hat{B} T))$ are $p$-complete. Each of these spaces is $p$-complete for the same reason: it is the total space in a fibration where the base and fiber are $p$-complete, and the fundamental group of the base acts trivially on the mod $p$ cohomology of the fiber [B-K, II, Lemma 5.1].

Now let $S=H^{*} \operatorname{Borel}(\pi, \hat{B} T)$ and recall the algebra $A_{W}$ can be obtained from $S$ by letting $W \subseteq G L_{n}\left(Z_{p}\right)$ act on $\hat{B} T$ and taking $A_{W}$ to be the elements of $S^{W}$ of degree greater than one. Because $T^{V}$ is exact, $T_{f}^{V} A_{W} \subseteq T_{f}^{V} S^{W}$. Since $T^{V}$ commutes with invariants, we can compute $T^{V} S^{W}$ by understanding the action that $G L_{n}\left(Z_{p}\right)$ has on $T^{V} S$ through its action on $S$. Similarly, the $G L(V)$ action on $T^{V} A_{W}$ is a restriction of the $G L(V)$ action on 
$T^{V} S$. The commutative ladder of Proposition 3.2 says that both of these actions can be obtained from studying the actions of $G L_{n}\left(Z_{p}\right)$ and $G L(V)$ on $\operatorname{map}(B V, \hat{B} T)$ (by acting on $\hat{B} T$ and $B V$, respectively), and hence on $\operatorname{map}(B V, \operatorname{Borel}(\pi, \hat{B} T)) \simeq \operatorname{Borel}(\pi, \operatorname{map}(B V, \hat{B} T))$. Note that the actions of $G L_{n}\left(Z_{p}\right)$ and $G L(V)$ on $\operatorname{map}(B V, \hat{B} T)$ commute with each other and with the action of $\pi$.

We return to the fact that

$$
\operatorname{map}(B V, \hat{B} T) \simeq \coprod_{\operatorname{End}(V)} \hat{B} T
$$

or alternatively, $\operatorname{map}(B V, \hat{B} T) \simeq \operatorname{End}(V) \times \hat{B} T$. (One can think of $\operatorname{End}(V)$ as indexing the possible maps $H^{*} \hat{B} T \rightarrow H^{*} B V$.) The action of $G L(V)$ simply permutes the coordinates, and so on cohomology an element $g \in G L(V)$ acts by

$$
g(f, x)=\left(f g^{-1}, x\right) \text { for }(f, x) \in \operatorname{End}(V) \times H^{*} \hat{B} T .
$$

An element $h \in G L_{n}\left(Z_{p}\right)$, on the other hand, acts both by changing the component and by twisting: if $\bar{h}$ denotes the reduction of $h \bmod p$, then

$$
(f, x) h=\left(\bar{h}^{-1} f, h^{*} x\right) .
$$

Proposition 3.4. Let $W \subseteq G L_{n}\left(Z_{p}\right)$ and for any $e: H^{*} \hat{B} T \rightarrow H^{*} B V$, let $W_{e}$ be the subgroup of $W$ which fixes $e$. Let $\bar{W}$ denote the reduction of $W$ mod p. Then

$$
T^{V}\left(H^{*} \operatorname{Borel}(\pi, \hat{B} T)\right)^{W} \cong \bigoplus_{\langle e\rangle \in \bar{W} \backslash \operatorname{End}(V)}\left(H^{*} \operatorname{Borel}(\pi, \hat{B} T)\right)^{W_{e}} .
$$

The action of $G L(V)$ on $T^{V}\left(H^{*} \operatorname{Borel}(\pi, \hat{B} T)\right)^{W}$ is by

$$
g(h, x)=\left(h g^{-1},\left(g^{-1}\right)^{*} x\right) .
$$

Corollary 3.5.

$$
T_{f}^{V}\left(H^{*} \operatorname{Borel}(\pi, \hat{B} T)\right)^{W} \cong H^{*} \operatorname{Borel}(\pi, \hat{B} T)
$$

where $\bar{w} \in \bar{W} \subseteq G L(V)$ acts on $H^{*} \operatorname{Borel}(\pi, \hat{B} T)$ by the map

$$
\left(w^{-1}\right)^{*}: H^{*} \operatorname{Borel}(\pi, \hat{B} T) \rightarrow H^{*} \operatorname{Borel}(\pi, \hat{B} T) .
$$

The proofs of Proposition 3.4 and Corollary 3.5 are easy calculations using the formulas above for the action of $G L_{n}\left(Z_{p}\right)$ and $G L(V)$ on $H^{*} \operatorname{Borel}(\pi, \hat{B} T)$. Corollary 3.5 uses the fact that the component $T_{f}^{V} H^{*} \operatorname{Borel}(\pi, \hat{B} T)$ corresponds to the coset of the identity element of $\operatorname{End}(V)$.

Finally, we must extract $T_{f}^{V} A_{W}$ from $T^{V}\left(H^{*} \operatorname{Borel}(\pi, \hat{B} T)\right)^{W}$.

Proposition 3.6. The inclusion $A_{W} \rightarrow\left(H^{*} \operatorname{Borel}(\pi, \hat{B} T)\right)^{W}$ induces a mapping $T^{V} A_{W} \rightarrow T^{V}\left(H^{*} \operatorname{Borel}(\pi, \hat{B} T)\right)^{W}$ which is an isomorphism an all components except the zero component.

Proof. We first recall that for any algebra $K \in \mathscr{K}$ with an finite set of algebra indecomposables, the null component of $T^{V} K$ is isomorphic to $K$ [D-W, Theorem 3.2]. In this case the map $\eta_{K}: T^{V} K \rightarrow K$ which is adjoint to 
$K \rightarrow H^{*} B V \otimes K$ by $k \rightarrow(1, k)$ is an isomorphism from the null component of $T^{V} K$ to $K$. The kernel of $\eta_{K}$ is then isomorphic to the sum of the non-null components of $T^{V} K$.

Now consider the following ladder of exact sequences:

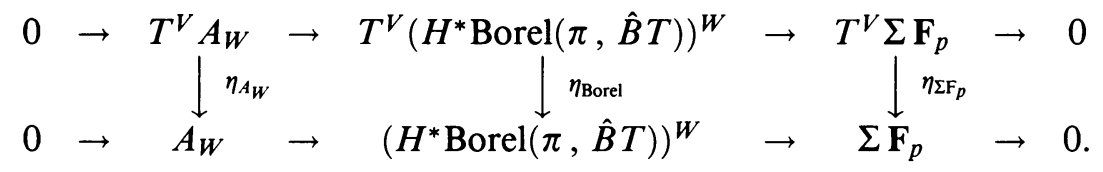

The result now follows from the observations above, the fact that $\eta_{\Sigma \mathrm{F}_{p}}$ is an isomorphism [Lannes, Proposition 2.2.4], and the fact that $A_{W}$ and $\left(H^{*} \operatorname{Borel}(\pi, \hat{B} T)\right)^{W}$ are finitely generated algebras.

Corollary 3.7.

$$
T_{f}^{V}\left(A_{W}\right) \cong H^{*} \operatorname{Borel}(\pi, \hat{B} T)
$$

\section{4. Сономоlogy of $\operatorname{map}(B V, Y)_{f}$}

The goal of this section is to prove the following proposition.

Proposition 4.1. Let $Y$ be a space with $H^{*} Y \cong A_{W}$, and let $f: H^{*} Y \rightarrow H^{*} B V$ be the inclusion of invariants on even dimensions. Then $H^{*} \operatorname{map}(B V, Y)_{f} \cong$ $T_{f}^{V} H^{*} Y$.

The proof of Proposition 4.1 is at the end of the section. In the next few paragraphs we outline the strategy, which is the same as that of [ABN, Section 6].

First note that the isomorphism of Proposition 4.1 is not guaranteed to us by the usual $T$-technology [Lannes, Corollary 3.2.2] because $T_{f}^{V} H^{*} Y$ is not zero in dimension one. Nonetheless, the work of Dror Farjoun and Smith shows that $T_{f}^{V} H^{*} Y$ always has a geometric interpretation, even when it does not give the cohomology of the mapping space. We write $P_{n} Y$ for the $n$th Postnikov section of $Y$, and $f_{n}$ for the composite $B V \rightarrow Y \rightarrow P_{n} Y$. Farjoun and Smith [DF-S, Theorem 1.1] show

$$
T_{f}^{V} H^{*} Y \cong \underset{n}{\lim } H^{*} \operatorname{map}\left(B V, P_{n} Y\right)_{f_{n}} .
$$

(To apply their theorem we use the fact that $Y$ is $p$-complete and nilpotent, the latter following from simple connectivity of $Y$.) There is an obvious map

$$
\underset{n}{\lim _{n}} H^{*} \operatorname{map}\left(B V, P_{n} Y\right)_{f_{n}} \rightarrow H^{*} \underbrace{\lim }_{n} \operatorname{map}\left(B V, P_{n} Y\right)_{f_{n}},
$$

and the content of Proposition 4.1 is that this map is an isomorphism even though, in general, cohomology is poorly related to inverse limits of spaces.

We proceed by making the spaces $\operatorname{map}\left(B V, P_{n} Y\right)_{f_{n}}$ total spaces of fibrations whose bases and fibers turn out to be tractable. By Corollary 3.7, we know $T_{f}^{V} H^{*} Y \cong E(z) \otimes \mathbf{F}_{p}\left[x_{1}, \ldots, x_{n}\right]$ where $\beta x_{j}=z x_{j}$. The class $z \in$ $T_{f}^{V} H^{*} Y \cong \lim _{n} H^{1} \operatorname{map}\left(B V, P_{n} Y\right)_{f_{n}}$ cannot support any Bockstein (all classes in dimension two support a first order Bockstein), and therefore it must come 
from a sequence of compatible classes $z_{n} \in H^{1} \operatorname{map}\left(B V, P_{n} Y\right)_{f_{n}}$ on which higher and higher order Bocksteins are defined. We can choose compatible maps $\operatorname{map}\left(B V, P_{n} Y\right)_{f_{n}} \rightarrow K\left(Z / p^{\alpha(n)}, 1\right)$ with $\alpha(n) \rightarrow \infty$ as $n \rightarrow \infty$, and we write $F_{n}$ for the fiber of $\operatorname{map}\left(B V, P_{n} Y\right)_{f_{n}} \rightarrow K\left(Z / p^{\alpha(n)}, 1\right)$. This gives a map of towers

$$
\left\{F_{n}\right\} \rightarrow\left\{\operatorname{map}\left(B V, P_{n} Y\right)_{f_{n}}\right\} \rightarrow\left\{K\left(Z / p^{\alpha(n)}, 1\right)\right\}
$$

and a fibration at the inverse limit

$$
\underset{n}{\lim _{n}} F_{n} \rightarrow \operatorname{map}(B V, Y)_{f} \rightarrow K(\pi, 1) .
$$

(Recall that $\pi \cong Z_{p}$.) It turns out that circumstances are favorable for computing $\lim _{n} H^{*} F_{n}$ and for showing that $H^{*}{\underset{\lim }{\longleftarrow}}_{n} F_{n} \cong \lim _{n} H^{*} F_{n}$. Further, $H^{*} K(\pi, 1) \cong \lim _{n} H^{*} K\left(Z / p^{\alpha(n)}, 1\right)$. Our plan is to compare the Serre spectral sequence for the inverse limit fibration with the direct limit of the Serre spectral sequences for each $n$ to give $H^{*} \lim _{n} \operatorname{map}\left(B V, P_{n} Y\right)_{f_{n}}$.

Lemma 4.2. $\lim _{n} H^{*} F_{n} \cong \mathbf{F}_{p}\left[x_{1}, \ldots, x_{n}\right]$.

Proof. For each $n$, the fibration

$$
F_{n} \rightarrow \operatorname{map}\left(B V, P_{n} Y\right)_{f_{n}} \rightarrow K\left(Z / p^{\alpha(n)}, 1\right)
$$

has a converging Eilenberg-Moore spectral sequence, since the fundamental group of the base is a finite $p$-group [Dwyer]. Hence for each $n$ we have a spectral sequence

$$
\operatorname{Tor}_{H^{*} B Z / p^{\alpha(n)}}\left(H^{*} \operatorname{map}\left(B V, P_{n} Y\right)_{f_{n}}, \mathbf{F}_{p}\right) \Rightarrow H^{*} F_{n} .
$$

We would like to take the direct limit over $n$ of these spectral sequences, but we note that in general the direct limit of spectral sequences need not converge to the direct limit of the abutments. The two possible problems are (1) nondetection: it could be that an element of the direct limit of the abutments moves into higher and higher filtration as it moves through the direct limit of spectral sequences, in which case it would not be detected in the direct limit spectral sequence; and (2) fake cycles: if an element supports longer and longer differentials as it goes through the direct limit, it will be an infinite cycle in the direct limit spectral sequence even though it does not represent a class in the abutment at any finite stage. In the case of the cohomology Eilenberg-Moore spectral sequence, however, neither of these things can happen. The filtration giving rise to the spectral sequence is

$$
\cdots \supseteq F_{-2} \supseteq F_{-1} \supseteq F_{0}
$$

and the differentials raise filtration, so any given spot in the spectral sequence can only support differentials of fixed length. Further, a class can only move up in filtration a finite number of times as it goes through the direct limit, taking care of the non-detection problem. 
Hence we can pass to the direct limit over $n$ and obtain a converging direct limit spectral sequence

$$
\operatorname{Tor}_{E(z)}\left(E(z) \otimes \mathbf{F}_{p}\left[x_{1}, x_{2}, \ldots, x_{n}\right], \mathbf{F}_{p}\right) \Rightarrow \underset{n}{\lim } H^{*} F_{n} .
$$

The direct limit spectral sequence collapses and we find

$$
\underset{\lim }{\longrightarrow} H^{*} F_{n} \cong \mathbf{F}_{p}\left[x_{1}, x_{2}, \ldots, x_{n}\right]
$$

Lemma 4.3. $H^{*} \lim _{n} F_{n} \cong \lim _{n} H^{*} F_{n}$.

Lemma 4.3 follows from the following lemma of Lannes [Lannes, Lemme 3.2.3], for which we recall some definitions. A tower of groups $\left\{G_{n}\right\}$ is protrivial if for each $n$ there exists a $k$ such that $G_{n+k} \rightarrow G_{n}$ is the zero map. A map between towers of groups is a proisomorphism if the towers of kernels and cokernels are protrivial, and a tower is proconstant if it is proisomorphic to a constant tower.

Lemma 4.4. Let $\left\{Z_{n}\right\}$ be a tower of fibrations of pointed spaces and let $Z_{\infty}$ be the inverse limit of the tower. We make the following hypotheses:

(1) The tower $\left\{\bar{H}_{0} Z_{n}\right\}$ is protrivial.

(2) The tower of groups $\left\{\pi_{1} Z_{n}\right\}$ is proisomorphic to a finite p-group $P$.

(3) The tower of $\mathbf{F}_{p}$-vector spaces $\left\{H_{i} Z_{n}\right\}$ is proconstant for $i \geq 2$.

Then the map $\bar{H}_{i} Z_{\infty} \rightarrow\left\{\bar{H}_{i} Z_{n}\right\}$ is a proisomorphism for each $i$ and the map $\pi_{1} Z_{\infty} \rightarrow\left\{\pi_{1} Z_{n}\right\}$ induces an isomorphism $\pi_{1} Z_{\infty} \cong P$.

Proof of Lemma 4.3. We verify that the conditions of Lemma 4.4 are met in the case of the tower $\left\{F_{n}\right\}$. We have to convert our information about cohomology into information about homology, for which we need to prove finiteness. However, having established that, we will use the fact that if $\left\{G_{n}\right\}$ is a tower of finite groups, then

(1) $\lim _{n} G_{n}=0 \Rightarrow\left\{G_{n}\right\}$ is protrivial.

(2) $\varlimsup_{\lim _{n}} G_{n}$ finite $\Rightarrow\left\{G_{n}\right\}$ is proconstant.

We first show that $H_{i} F_{n}$ is finite. Our $p$-complete space $Y$ with $H^{*} Y \cong A_{W}$ has homotopy groups which are finite $p$-groups, by the same argument as that of Proposition 2.3, which depends only on cohomology. An inductive argument shows that $\pi_{i} \operatorname{map}\left(B V, P_{n} Y\right)_{f_{n}}$ is a finite $p$-group for all $i$ and $n$, and therefore $\pi_{i} F_{n}$ is also a finite $p$-group by the long exact sequence in homotopy for the fibration

$$
F_{n} \rightarrow \operatorname{map}\left(B V, P_{n} Y\right)_{f_{n}} \rightarrow K\left(Z / p^{\alpha(n)}, 1\right) .
$$

Therefore $F_{n}$ is $p$-complete, nilpotent, and $H^{*} F_{n}$ is of finite type. Now we check the hypotheses of Lemma 4.4, using the fact that $H_{*} F_{n}$ is finite and dual to $H^{*} F_{n}$.

(1) $\lim _{n} \bar{H}^{0} F_{n}=0 \Rightarrow \lim _{n} \bar{H}_{0} F_{n}=0 \Rightarrow\left\{\bar{H}_{0} F_{n}\right\}$ is protrivial.

(2) We claim that the tower $\left\{\pi_{1} F_{n}\right\}$ is actually protrivial. First, $\lim _{n} H^{1} F_{n}=$ $0 \Rightarrow \lim _{n} H_{1} F_{n}=0$ and the tower $\left\{H_{1} F_{n}\right\}$ is protrivial. Therefore for 
each $n$ there exists $N$ such that the image of the map $\pi_{1} F_{n+N} \rightarrow \pi_{1} F_{n}$ is contained in the commutator subgroup of $\pi_{1} F_{n}$. However, $\pi_{1} F_{n}$ is a finite $p$-group and must be nilpotent. An inductive argument then shows that for some $N^{\prime}$, the map $\pi_{1} F_{n+N^{\prime}} \rightarrow \pi_{1} F_{n}$ is actually zero.

(3) $\lim _{n} H^{i} F_{n}$ is finite, so $\lim _{n} H_{i} F_{n}$ is finite and $\left\{H_{i} F_{n}\right\}$ is proconstant.

Therefore Lemma 4.4 applies to give us $H_{*} \lim _{n} F_{n} \cong \lim _{n} H_{*} F_{n}$, and dually, the conclusion of Lemma 4.3.

Hence we know that $H^{*} \lim _{n} F_{n} \cong \mathbf{F}_{p}\left[x_{1}, x_{2}, \ldots, x_{n}\right]$, and we would like to conclude that $\lim _{n} F_{n} \simeq \hat{B} T$. There is certainly a map $\longleftarrow_{n} F_{n} \rightarrow \hat{B} T$ which is a mod $p$ homology isomorphism. To know that this map is a homotopy equivalence, it is then sufficient to know that both spaces are $H \mathbf{F}_{p}$-local, which is certainly true for $\hat{B} T$. In the case of $\lim _{n} F_{n}$, note that since $Y$ is $p$-complete, it is $H \mathbf{F}_{p}$-local, and hence $\operatorname{map}(B V, Y)_{f}$, being the homotopy (inverse) limit of local spaces, is again local. Then $\lim _{n} F_{n}$ is the homotopy fiber of a map between the local spaces $\operatorname{map}(B V, Y)_{f}$ and $K(\pi, 1)$ and is local also, finishing the proof that $\lim _{n} F_{n} \simeq \hat{B} T$. Thus the inverse limit fibration of

$$
\left\{F_{n}\right\} \rightarrow\left\{\operatorname{map}\left(B V, P_{n} Y\right)_{f_{n}}\right\} \rightarrow\left\{K\left(Z / p^{\alpha(n)}, 1\right)\right\}
$$

is a fibration

$$
\hat{B} T \rightarrow \operatorname{map}(B V, Y)_{f} \rightarrow B \pi .
$$

Proof of Proposition 4.1. We compare Serre spectral sequences using the maps of fibrations

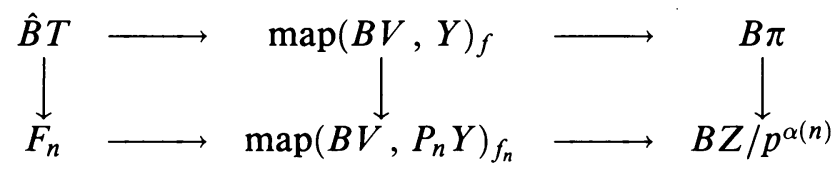

We must use local coefficients, since the actions of the fundamental groups of the bases on the cohomology of the fibers are not known to be trivial. We obtain compatible maps of spectral sequences

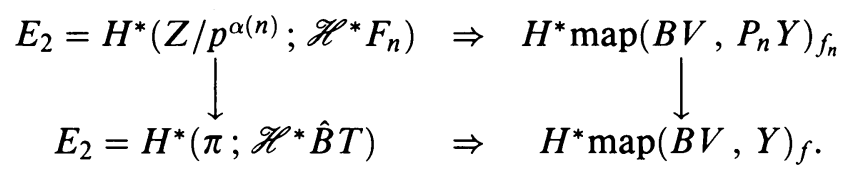

As in the proof of Lemma 4.2, we can pass to the direct limit to obtain a map of spectral sequences

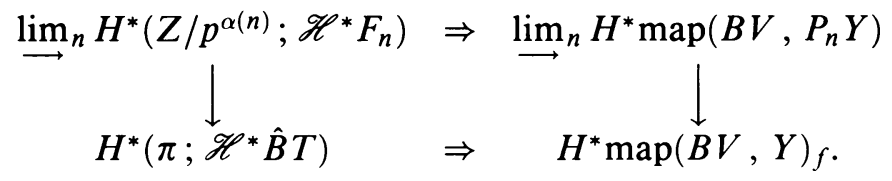


This map of spectral sequences is an isomorphism on $E_{2}$ terms by Lemma 4.5 below, because $H^{*} \hat{B} T \cong \lim _{n} H^{*} F_{n}$, and therefore

$$
\begin{aligned}
H^{*} \operatorname{map}(B V, Y)_{f} & \cong \lim _{n} H^{*} \operatorname{map}\left(B V, P_{n} Y\right) \\
& \cong T_{f}^{V} H^{*} Y \\
& \cong E(z) \otimes \mathbf{F}_{p}\left[x_{1}, x_{2}, \ldots x_{n}\right]
\end{aligned}
$$

and the proof of Lemma 4.1 is complete.

Lemma 4.5. Let $M_{n}$ be a modp vector space which is a module over $Z\left[Z / p^{n}\right]$ and suppose given maps $M_{n} \rightarrow M_{n+1}$ which are $Z / p^{n+1}$-equivariant. If $\stackrel{\lim }{\longrightarrow} M_{n}$ is a finite vector space, then

$$
H^{*}\left(Z_{p} ; \lim _{\longrightarrow} M_{n}\right) \cong \lim _{\longrightarrow} H^{*}\left(Z / p^{n} ; M_{n}\right) .
$$

Proof. By direct computation with [Brown, p. 58], one can show that if $M$ is a $Z\left[Z / p^{n}\right]$-module which is all $p$-torsion, then

$$
\underset{i}{\lim } H^{*}\left(Z / p^{n+i} ; M\right) \cong H^{*}(Z ; M) .
$$

Hence using the fact that $H^{*}\left(Z ; \underset{\lim }{\longrightarrow} M_{n}\right) \cong \underset{\lim }{\longrightarrow}\left(Z ; M_{n}\right)$, under the hypotheses of the lemma we have

$$
H^{*}\left(Z ; \lim _{\longrightarrow} M_{n}\right) \cong \lim _{\longrightarrow} H^{*}\left(Z / p^{n} ; M_{n}\right) .
$$

On the other hand, by considering the Serre spectral sequence for $Z \rightarrow Z_{p} \rightarrow$ $Z_{p} / Z$, we can see that if $\lim _{\longrightarrow} M_{n}$ is a finite $\bmod p$ vector space, then

$$
H^{*}\left(Z ; \lim _{\longrightarrow} M_{n}\right) \cong H^{*}\left(Z_{p} ; \underset{\lim }{\longrightarrow} M_{n}\right),
$$

the point being that the uniquely $p$-divisible group $Z_{p} / Z$ must have trivial action on the finite vector space $H^{*}\left(Z ; \lim M_{n}\right)$, and $H^{i}\left(Z_{p} / Z ; Z / p\right)=0$ for $i>0$.

\section{5. НомотоРY TYPE OF $\operatorname{map}(B V, Y)_{f}$}

In the previous section we showed that $\operatorname{map}(B V, Y)_{f}$ lies in a fibration

$$
\hat{B} T \rightarrow \operatorname{map}(B V, Y)_{f} \rightarrow B \pi .
$$

In this section we identify the homotopy type of $\operatorname{map}(B V, Y)_{f}$. We first note that such fibrations are classified by

(1) An action of $\pi$ on $Z_{p}^{n}$, and

(2) An element of $H^{3}\left(\pi ; Z_{p}^{n}\right)$.

Since the cohomology group is zero, $\operatorname{map}(B V, Y)_{f}$ is determined by the action of $\pi$ on $Z_{p}^{n}$, which we calculate in this section.

Proposition 5.1. If $Y$ is p-complete with $H^{*} Y \cong A_{W}$ where $W$ is an irreducible finite pseudoreflection group of order prime to $p$, then $\operatorname{map}(B V, Y)_{f} \simeq$ $\operatorname{Borel}(\pi, \hat{B} T)$. 
Remark. It is easy to see from the Serre spectral sequence (with twisted coefficients) for the fibration

$$
\hat{B} T \rightarrow \operatorname{map}(B V, Y)_{f} \rightarrow B \pi
$$

that the action of $\pi$ on $H^{*} \hat{B} T$ must be trivial-otherwise the $E_{2}$-term would be too small to give $H^{*} \operatorname{map}(B V, Y)_{f} \cong E(z) \otimes \mathbf{F}_{p}\left[x_{1}, \ldots, x_{n}\right]$, as computed in the previous section. However, we note in passing that cohomology is NOT sufficient to determine the homotopy type of the $\operatorname{space} \operatorname{map}(B V, Y)_{f}$.

Our strategy to prove Proposition 5.1 is to show that the action of $W$ on $\operatorname{map}(B V, Y)_{f}$ can be made to give an action of $W$ on $Z_{p}^{n}$ which commutes with the action of $\pi \cong \pi_{1} \operatorname{map}(B V, Y)_{f}$ on $Z_{p}^{n} \cong \pi_{2} \operatorname{map}(B V, Y)_{f}$. If we then assume that $W$ is an irreducible representation, a variation of Schur's Lemma tells us that $\pi$ must act by multiplication by scalars. Just what the scalars are is detected by the action of the Bockstein in $H^{*} \operatorname{map}(B V, Y)_{f}$.

The action of $W$ on $\operatorname{map}(B V, Y)_{f}$ does not automatically give an action of $W$ on $\pi_{2} \operatorname{map}(B V, Y)_{f} \cong Z_{p}^{n}$ because of basepoint problems. Instead, we consider the Borel construction for the action of $W$ on $\operatorname{map}(B V, Y)_{f}$ and show that its fundamental group, which is an extension of $W$ by $\pi$, is actually a direct product. This provides commuting actions of $W$ and $\pi$ on $\pi_{2} \operatorname{Borel}\left(W, \operatorname{map}(B V, Y)_{f}\right) \cong \pi_{2} \operatorname{map}(B V, Y)_{f} \cong Z_{p}^{n}$.

Proposition 5.2.

$$
\pi_{1} \operatorname{Borel}\left(W, \operatorname{map}(B V, Y)_{f}\right) \cong W \times \pi .
$$

Proof. The long exact homotopy sequence for the fibration

$$
\hat{B} T \rightarrow \operatorname{map}(B V, Y)_{f} \rightarrow B \pi
$$

gives $\pi_{1} \operatorname{map}(B V, Y)_{f} \cong \pi$. Therefore $\pi_{1} \operatorname{Borel}\left(W, \operatorname{map}(B V, Y)_{f}\right)$ is an extension

$$
0 \rightarrow \pi \rightarrow \pi_{1} \rightarrow W \rightarrow 1 .
$$

Such an extension is determined by an action of $W$ on $\pi$ and an element of $H^{2}(W ; \pi)$. Because $|W|$ is prime to $p$, we have $H^{*}(W ; \pi)=0$. Hence the extension is a semi-direct product, and is completely specified by a homomorphism $W \rightarrow \operatorname{Aut}(\pi) \cong Z /(p-1) \times Z_{p}$. Since $W$ is torsion, its image is contained in $Z /(p-1)$.

We will show that the homomorphism $W \rightarrow \operatorname{Aut}(\pi)$ is actually trivial. We know $\pi_{1} \operatorname{map}(B V, Y)_{f} \cong H_{1}\left(\operatorname{map}(B V, Y)_{f} ; Z\right) \cong \pi$, and the action of $W$ on $\pi$ in the extension $(*)$ is the same as the action of the fundamental group of the base on the one-dimensional homology of the fiber in the fibration

$$
\operatorname{map}(B V, Y)_{f} \rightarrow \operatorname{Borel}\left(W, \operatorname{map}(B V, Y)_{f}\right) \rightarrow B W .
$$

However, the action of $W$ on $H_{1}\left(\operatorname{map}(B V, Y)_{f} ; \mathbf{F}_{p}\right)$ is trivial, by Corollary 3.5 and Proposition 3.6. Since any nontrivial action of a finite group on $\pi$ would still be detected after reduction to $Z / p$, the action of $W$ on $\pi$ must actually be trivial. Therefore the extension $(*)$ is a semi-direct product with trivial action, i.e. $\pi_{1} \operatorname{Borel}\left(W, \operatorname{map}(B V, Y)_{f}\right) \cong W \times \pi$. 
Proof of Proposition 5.1. We know that the homotopy type of $\operatorname{map}(B V, Y)_{f}$ is determined by the action of the fundamental group, $\pi$, on $\pi_{2} \operatorname{map}(B V, Y)_{f} \cong$ $Z_{p}^{n}$ : any such action corresponds to a unique space (up to homotopy), namely the Borel construction for the associated action of $\pi$ on $\hat{B} T$. Hence to compute the homotopy type of $\operatorname{map}(B V, Y)_{f}$, we need only determine the action of $\pi_{1}$ on $\pi_{2}$.

To analyze the action of $\pi$ on $Z_{p}^{n}$, consider the fibration

$$
\operatorname{map}(B V, Y)_{f} \rightarrow \operatorname{Borel}\left(W, \operatorname{map}(B V, Y)_{f}\right) \rightarrow B W .
$$

Inclusion of the fiber gives a monomorphism $\pi \rightarrow W \times \pi$ on the fundamental group, and an isomorphism on $\pi_{2}$, so the action of $\pi \subseteq \pi_{1}$ on $\pi_{2} \cong Z_{p}^{n}$ is the same in the fiber and total space. On the other hand, in the total space, we have an action of $W \times \pi$ on $Z_{p}^{n}$, that is, we have commuting actions of $W$ and $\pi$ on $Z_{p}^{n}$. Now the representation of $W$ on $Z_{p}^{n}$ is, up to conjugacy, the natural one given by the fact that $W$ is a subgroup of $G L_{n}\left(Z_{p}\right)$, for that is certainly what it is on homology (see Corollary 3.5) and by [DMW, Theorem 1.5], a pseudoreflection group of order prime to $p$ in $G L_{n}\left(\mathbf{F}_{p}\right)$ lifts uniquely up to conjugacy to a subgroup of $G L_{n}\left(Z_{p}\right)$.

However, by assumption we have an irreducible representation of $W$. If we were studying representations over complex numbers, Schur's Lemma would say that the action of $\pi$ had to be multiplication by scalars. We imitate the proof of Schur's Lemma in the $p$-adic context. Tensoring $Z_{p}^{n}$ with the rationals gives us a representation of $W \times Z_{p}$ over $Q_{p}$. The only endomorphisms of this representation of $W$ are either automorphisms or zero, since the representation is irreducible. Hence the action of an element $z \in \pi$ on $Q_{p}^{n}$ is either zero or an automorphism. By assumption, $W$ is a pseudoreflection group, so $W$ contains at least one pseudoreflection, which has a one-dimensional eigenspace in $\left(Q_{p}\right)^{n}$. Since the action of $z \in Z_{p}$ must stabilize this eigenspace, $z$ must act there by multiplication by a scalar, say by multiplication by $c \in Q_{p}$. But then $z-c$ is an endomorphism of the representation of $W$ and has a nontrivial kernel, implying $z-c$ must be zero. Therefore $z$ acts by multiplication by $c$ on all of $\left(Q_{p}\right)^{n}$. Further, since the action of $z$ must preserve $\left(Z_{p}\right)^{n} \subseteq\left(Q_{p}\right)^{n}$, we must have $c \in Z_{p}$.

Therefore the action of $\pi$ on $Z_{p}^{n}$ is given by multiplication by scalars, that is, by a homomorphism $\pi \rightarrow G L_{n}\left(Z_{p}\right)$ whose image is multiples of the identity matrix. We can also think of this homomorphism as a map $\pi \rightarrow Z_{p}$ whose image lies in the $p$-adic units. Two such homomorphisms $f, g$ will give homotopy equivalent $\operatorname{Borel}$ constructions $\operatorname{Borel}(\pi, \hat{B} T)$ if there is an automorphism $\alpha$ of $\pi$ with $f=g \circ \alpha$. This will happen if the highest powers of $p$ dividing $1-f(1)$ and $1-g(1)$ are the same. Thus up to equivalence of the Borel construction, there is one action of $\pi$ for each positive integer, say by multiplication by $1+p^{k}$. However, a computation with the Serre spectral sequence with twisted coefficients shows that the Borel construction for multiplication by $1+p^{k}$ has a $k$ th order Bockstein on $H^{2}\left(\operatorname{Borel}(\pi, \hat{B} T) ; \mathbf{F}_{p}\right)$. Hence the fact that elements of $H^{2} \operatorname{map}(B V, Y)_{f}$ support a first order Bockstein implies that $\operatorname{map}(B V, Y)_{f} \simeq \operatorname{Borel}(\pi, \hat{B} T)$ where $\pi$ acts by multiplication by $1+p$ i.e. by the usual action of $\pi \subseteq G L_{1}\left(Z_{p}\right)$ on $Z_{p}$. 
Proof of Theorem 1.1. Let $Y$ be a $p$-complete space with $H^{*} Y \cong A_{W}$ and let $V$ be an elementary abelian $p$-group with rank equal to the number of polymonial generators of $A_{W}$. Let $f: A_{W} \rightarrow H^{*} B V$ be the map which is inclusion of invariants on even dimensions (and zero on odd ones) and also the function $B V \rightarrow Y$ which realizes it. By Proposition 5.1, $\operatorname{map}(B V, Y)_{f} \simeq \operatorname{Borel}(\pi, \hat{B} T)$, where $\pi$ acts on $\hat{B} T \simeq K\left(Z_{p}^{n}, 2\right)$ diagonally by multiplication by $1+p$. There is a continuous function

$$
\operatorname{map}(B V, Y)_{f} \rightarrow Y
$$

by basepoint evaluation, and this map is equivariant with respect to the action of the group $W \subseteq G L_{n}\left(Z_{p}\right)$ where the action on $Y$ is trivial and the action of $W$ on $\operatorname{map}(B V, Y)_{f}$ is through reduction of $W$ to $G L(V)$. (This is because the basepoint of $B V$ is fixed by the action of $W$ on $B V$.) This gives us an extension over the Borel construction

$$
\operatorname{Borel}\left(W, \operatorname{map}(B V, Y)_{f}\right) \rightarrow Y,
$$

and it is easy to see from Corollary 3.7 that this last map is an isomorphism on cohomology in dimensions greater than one. Further,

$$
\operatorname{Borel}\left(W, \operatorname{map}(B V, Y)_{f}\right) \simeq \operatorname{Borel}(W \times \pi, \hat{B} T),
$$

so what we really have is a map

$$
\operatorname{Borel}(W \times \pi, \hat{B} T) \rightarrow Y
$$

which is a cohomology isomorphism in dimensions greater than one.

As in the construction of $X_{W}$ in Section 2, let $g: S^{1} \rightarrow \operatorname{Borel}(W \times \pi, \hat{B} T)$ represent the element $\mathrm{id} \times 1 \in \pi_{1} \operatorname{Borel}(W \times \pi, \hat{B} T) \cong W \times Z_{p}$ and let $C_{W}$ be the cofiber of $g$. The composite

$$
S^{1} \rightarrow \operatorname{Borel}(W \times \pi, \hat{B} T) \rightarrow Y
$$

is null, because $Y$ is simply connected ( $p$-complete with $H^{1}=0$ ), and this gives an extension of $g$ over the cofiber, i.e. a map $C_{W} \rightarrow Y$ which is a cohomology isomorphism. But now notice that $Y$, being $p$-complete, must be the $p$-completion of $C_{W}$, i.e. $Y \simeq X_{W}$ as we set out to prove.

\section{REFERENCES}

[ABN] J. Aguade, C. Broto, and D. Notbohm, Homotopy classification of spaces with interesting cohomology and a conjecture of Cooke. I, Topology 33 (1994) 455-492.

[B-K] A. Bousfield and D. Kan, Homotopy limits, completions, and localizations, Lecture Notes in Math., vol. 304, Springer-Verlag, Berlin and New York, 1972.

[Brown] K. Brown, Cohomology of groups, Graduate Texts in Math., vol. 87, Springer-Verlag, New York, 1982.

[C-E] A. Clark and J. Ewing, The realization of polynomial algebras as cohomology rings, Pacific J. Math. 50 (1974), 425-434.

[DF-S] E. Dror Farjoun and J. Smith, A geometric interpretation of Lannes' functor T, Astérisque 191 (1990), 87-95.

[Dwyer] W. Dwyer, Strong convergence of the Eilenberg-Moore spectral sequence, Topology 13 (1974), 255-265.

[DMW] W. Dwyer, H. Miller, and C. Wilkerson, Homotopical uniqueness of classifying spaces, Topology 31 (1992), 29-45. 
[D-W] W. Dwyer and C. Wilkerson, Spaces of null homotopic maps, Proceedings of the 1988 Luminy Conference, Astérisque 191 (1990), 97-108.

[D-Z] - W. Dwyer and A. Zabrodsky, Maps between classifying spaces, Proceedings of the 1986 Barcelona Conference on Algebraic Topology, J. Aguade, and R. Kane, eds., Lecture Notes in Math., vol. 1298, Springer-Verlag, Berlin and New York, 1988.

[Lannes] J. Lannes, Sur les espaces fonctionels dont la source est le classifiant d'un p-groupe abelien elémentaire, Publ. Math. Inst. Hautes Etudes Sci. 75 (1992), 135-244.

Department of Mathematics, University of Toledo, Toledo, Ohio 43606-3390

E-mail address: klesh@uof t02.utoledo.edu 